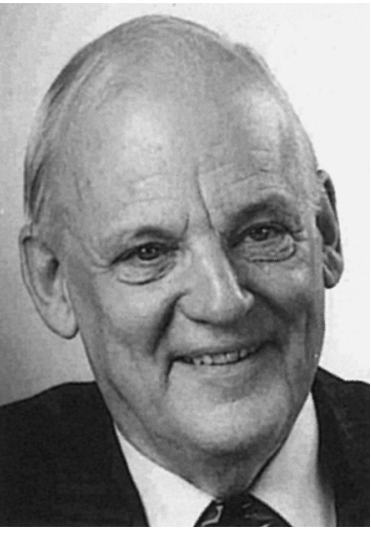

Gösta Samuelson gosta.samuelson@htu.se

\title{
Meal patterns impact and obesity
}

$\mathrm{D}$ uring recent decades meal patterns have changed in both adults and children. In a review article in this issue of Scandinavian Journal of Nutrition (SJN), France Bellisle states that in many developed countries traditional meal patterns have been lost. Snacking occurs at any time, and food is available around the clock and in large amounts. Earlier consumption studies suggested an inverse relationship between daily meal number and obesity, but this has not been confirmed using the doubly labelled water technique to measure energy expenditure. Many studies show an underreporting of food intake, by about $20 \%$ in both adults and adolescents. People who eat breakfast tend to eat less during the day, while people who eat late eat more. Furthermore, the content of snacks is often energy rich, but in a Swedish study referred to in the review all types of intake in meals and snacks were more frequent in obese than in control women. The energy content of meals and snacks is certainly an important factor in the prevention and treatment of obesity, even though there is no consistent evidence between eating events and obesity. Further articles on the impact of eating habits on human metabolism, as well as on behaviour, will be published in forthcoming issues of SJN.

\section{Physical activity in a changing society}

In an invited overview Lars-Magnus Engström emphasizes that in modern society our daily lives have become less and less physically active. The combination of changed eating habits and reduced physical activity is an important factor in the increased prevalence of overweight and obesity. Institutionalizing, body image and exercise as a lifestyle are discussed in the article. It is of utmost importance to promote physical activity in schools, particularly for children and adolescents who are not interested in taking part in competitive sports. Adults as well as the elderly should be encouraged and motivated to take minimum daily exercise, walking, cycling, swimming or other kinds of activity, but not necessarily feel that they are engaged in a fitness programme. Increased physical activity combined with balanced food intake equalizing energy expenditure would effectively reduce the prevalence of overweight and obesity in children, adolescents and adults.

\section{Is dietary fish intake good for cardiovascular health?}

During the past few decades, studies have indicated that the dietary intake of fish and its content of omega-3 polyunsaturated fatty acids (n-3 PUFA), or solely the intake of n-3 PUFA derived from fish oils, has antiatherogenic, antithrombotic, anti-inflammatory and/or antiarrhythmic effects. In most studies, however, fish and fish oils are not separated, which makes it is difficult to analyse whether compounds in fish other than n-3 PUFA have beneficial effects on the heart. In a review article in this issue Ingrid Undeland et al. summarize recent data from both epidemiological and intervention studies on the effects of fish consumption on coronary vascular disease and coronary mortality. The compilation of epidemiological studies provides substantial evidence that, even at low intake levels, fish has a beneficial effect on cardiovascular disease and mortality. However, it is important to emphasize that evidence from intervention studies is still inconsistent. Such studies are needed, as well as more detailed studies of the fish muscle matrix content. The preparation of fish may also affect other compounds. n-3 PUFA may not be the only influential factor: other compounds in fish may also be important in cardiovascular mortality and morbidity. n-3 PUFA fish also contains protein and minerals of high nutritional value. It should be consumed at least once a week. 